$01,07,11$

\title{
Упругие свойства сплавов TiNi с памятью формы с различной термообработкой
}

\author{
(C) 3.А. Казей, В.В. Снегирев \\ Московский государственный университет им. М.В. Ломоносова, \\ Москва, Россия \\ E-mail: kazei@plms.phys.msu.ru
}

Поступила в Редакцию 12 февраля 2019 г.

В окончательной редакции 12 февраля 2019 г.

Принята к публикации 12 февраля 2019 г.

Проведены сравнительные экспериментальные исследования упругих характеристик коммерческих сплавов нитинол TiNi c памятью формы, подвергнутых различной термообработке, в интервале температур 80-300 K, включающем предмартенситный и мартенситный переходы. Обнаружено, что искажение кристаллической структуры при предмартенситном переходе в образце „as prepared“ сопровождаются выраженными аномалиями модуля Юнга и внутреннего трения в области температуры фазового перехода $T_{S} \approx 270 \mathrm{~K}$ как при нагревании, так и при охлаждении. После дополнительной термообработки температуры предмартенситного перехода при нагреве $T_{S u}$ и охлаждении $T_{S d}$ немного смещаются и гистерезис увеличивается, а характер упругих аномалий практически не меняется. Наоборот, для мартенситного перехода при $T \sim 220 \mathrm{~K}$ аномалии упругих свойств наблюдаются только при охлаждении, а температура и характер аномалий претерпевают значительные изменения после термообработки.

Ключевые слова: структурные фазовые переходы, мартенситные переходы, сплавы с памятью формы, модуль Юнга, внутреннее трение, гистерезис.

DOI: 10.21883/FTT.2019.07.47828.380

\section{1. Введение}

Сплавы на основе железа, меди, кобальта, титана часто обнаруживают целую последовательность фазовых превращений, сопровождающихся изменением структуры, наиболее интригующими из которых является мартенситные превращения. Мартенситные переходы в этих сплавах были открыты и исследованы достаточно давно [1], однако до сих пор остается открытым целый ряд вопросов по механизму и кинетике этих превращений и особенностям физических свойств в различных фазах сплава.

Различные сплавы с мартенситными переходами, например титан-никелевые, представляют большой интерес благодаря эффекту памяти формы, т.е. их способности при нагревании полностью восстанавливать форму после пластической деформации, возникающей при прямом мартенситном переходе под воздействием механической нагрузки. Ответственным за это поведение является термоупругое фазовое превращение, которое происходит из упорядоченной объемно-центрированной структуры (называемой В2, аустенит или $\beta$-фаза) к термоупругой мартенситной фазе (B19' или $\alpha^{\prime \prime}$-фаза). Это превращение обуславливает интересные и практически важные свойства, такие как суперэластичность, суперпластичность, высокая демпфирующая способность и отличные износостойкость и коррозионная стойкость.

Фазовые переходы в сплавах с памятью формы исследовались различными методами, включая структурные, транспортные, акустические, магнитные и другие [2-5].
Анализ и интерпретация результатов для сплавов с мартенситными переходами осложняются тем, что на его температуру, тип, характер, кинетику и физические свойства сильно влияют состав, наличие легирующих элементов, микроструктура и другие факторы. В настоящее время акцент в исследовании переместился в область материаловедения, т.е. выявление условий получения сплавов с заданными характеристиками для конкретных применений [6-9] и изучение их поведения в специальных условиях [10]. Для снижения времени и стоимости разработок при создании новых сплавов Моринага и соавторы предложили расчетный метод на основе молекулярных орбиталей [11]. При прогнозировании стабильной фазы сплава и для учета влияния легирующих элементов используется карта устойчивости фаз как функции двух квантовых параметров: порядок связи $B_{0}$, характеризующей силу ковалентной связи между Ті и легирующим элементом, и уровень энергии орбитали $M_{d}$, связанный с электроотрицательностью и ионным радиусом элемента. С использованием этого метода было предложено и реализовано значительное число сплавов на основе $\mathrm{Ti}$ без $\mathrm{Ni}$ с различными температурами перехода аустенит-мартенсит и упругими характеристиками [6].

Образцы с памятью формы находят широкое практическое применение в различных областях техники, a также благодаря их биосовместимости в области биомедицины. Для практических применений важны как значения модуля Юнга и поглощения, так и их зависимости от температуры. Упругие свойства, модуль Юнга 
и внутреннее трение, которые очень чувствительны к фазовым переходам различной природы, позволяют надежно регистрировать переходы и изучать влияние на них различных факторов. В большинстве опубликованных ранее работ приводятся данные по статическому модулю упругости при комнатной температуре, определенному классическим методом из кривых (нагрузкадеформация) [6,7]. Имеется ряд работ, в которых измеряются динамические упругие свойства в основном методом импульс-эхо на частотах $2-5 \mathrm{MHz}$ [3,12-14]. Настоящая работа посвящена экспериментальному исследованию резонансным методом на частоте $\sim 100 \mathrm{kHz}$ динамических упругих свойств коммерческих сплавов нитинол TiNi c памятью формы в широком интервале температур, включающем область мартенситного фазового перехода.

\section{2. Образцы и экспериментальная техника}

Исследования упругих свойств проводились на поликристаллических коммерческих сплавах $\mathrm{TiNi}$, содержащих равное атомное соотношение элементов (Nitinol). В работе приводятся данные для образов одной партии приготовления с различной термообработкой, включающей отжиг на воздухе и закалку. Образец 1 дополнительной термообработке не подвергался (,as prepared“), образцы 2 и 3 после отжига при $500^{\circ} \mathrm{C}$ закалялись в воду и на воздухе, соответственно, образец 4 - медленно охлаждался в печи (отпускался).

Модуль Юнга $E$ и коэффициент внутреннего трения $q^{-1}$ (обратная добротность) измерялись методом составного резонатора на частоте $\sim 110 \mathrm{kHz}$ в интервале температур 80-280 K. В автоматизированной установке в качестве задающего генератора использовался Agilent 33120A, в качестве регистрирующего устройства - цифровой осциллограф Tektronix TDS 1002 с дополнительным усилителем Stanford Research 560. Температура измерялась и контролировалась с помощью температурного контроллера Lakeshore 331 с точностью $\pm 0.03 \mathrm{~K}$. Температурные измерения проводились в режиме стационарного состояния, а температурный шаг и выдержка в области фазового перехода варьировались в широких пределах, обеспечивающих стационарность. Управление экспериментом и первичная обработка проводилась на стандартном IBM совместимом компьютере с интерфейсом IEEE-488 с использованием оригинального программного обеспечения. Из экспериментальной амплитудно-частотной характеристики $A(f)$ в области резонанса методом математической обработки получалась сглаженная кривая $A_{s m}(f)$ и определялись амплитуды и частоты резонанса и антирезонанса (ширина окна $\Delta f$ при сглаживании варьировалась в зависимости от качества исходных кривых $A(f))$. Температурные зависимости амплитуд и частот резонанса и антирезонанса позволяли определять изменение модуля Юнга и поглощения (коэффициента внутреннего трения) в широком диапазоне температур.

\section{3. Экспериментальные результаты и обсуждение}

\section{1. Модуль Юнга и внутреннее трение}

Обсудим сначала особенности поведения упругих свойств образца 1, не повергавшегося термообработке. На рис. $1, a, b$ приведены температурные зависимости относительной величины модуля Юнга $\Delta E(T) / E_{0}=\left[E(T) / E_{0}-1\right] \quad\left(\Delta E(T)=E(T)-E_{0}\right)$ $\left(E_{0}=E(T=300 \mathrm{~K})\right)$ и коэффициента внутреннего трения $q^{-1}(T)$ соответственно для этого образца. Для удобства сравнения зависимости $\Delta E(T) / E_{0}$ для всех образцов нормированы на их значение $E_{0}$ при $T=300 \mathrm{~K}$. Абсолютные величины $E_{0}$ образцов с различной термообработкой достаточно близки, мало меняются при термообработке и лежат в диапазоне $(7.13-7.15) \cdot 10^{11} \mathrm{din} / \mathrm{cm}^{2}$. Исключение составляет образец 4, у которого несколько меньшее значение $E_{0}=6.23 \cdot 10^{11} \mathrm{din} / \mathrm{cm}^{2}$ связано с близостью температуры перехода к $T=300 \mathrm{~K}$. Характер зависимостей

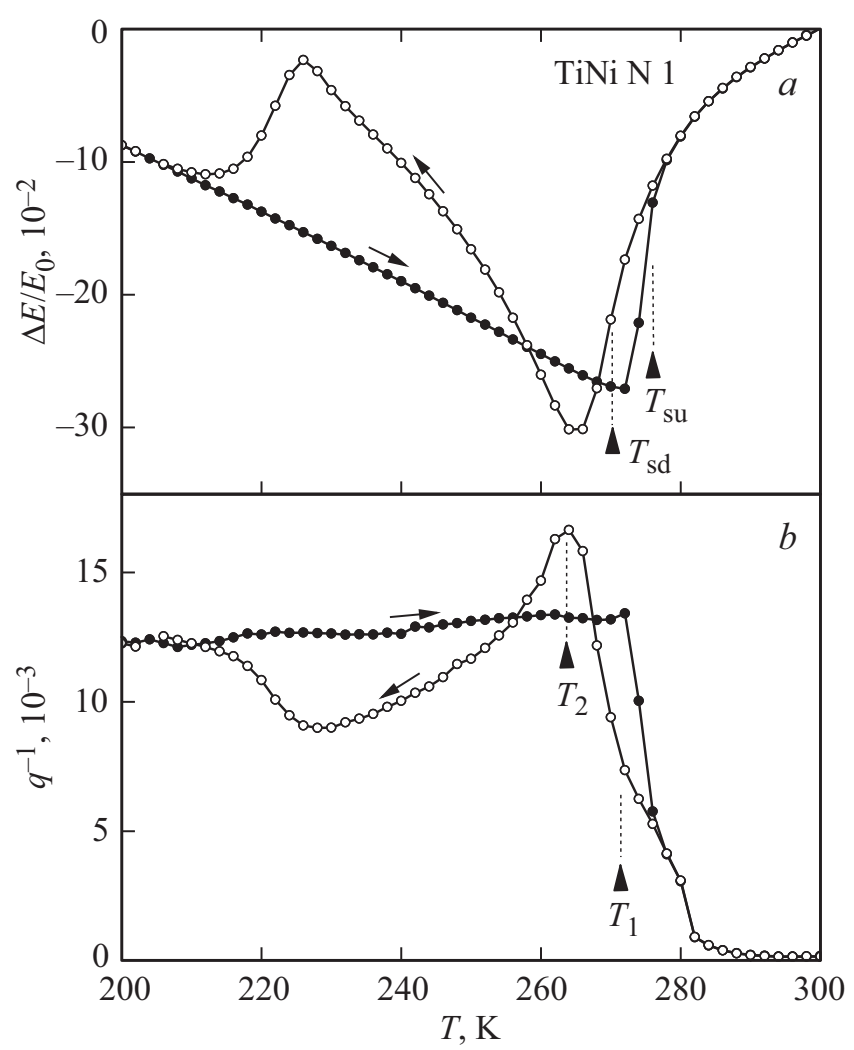

Рис. 1. Зависимость относительной величины модуля Юнга $\Delta E / E_{0}(a)$ и внутреннего трения $q^{-1}(T)(b)$ от температуры для образца 1 „as prepared“ сплава TiNi при нагреве (темные точки) и охлаждении (светлые точки). Температуры $T_{S u}$ и $T_{S d}$ соответствуют скачку модуля Юнга при нагреве и охлаждении, соответственно (см. текст). 
$\Delta E(T) / E_{0}$ в интервале $150-300 \mathrm{~K}$ при нагревании и охлаждении различен и зависит от скорости изменения температуры. На рис. 1 и далее приведены воспроизводимые кривые, полученные при медленном прохождении этого температурного интервала.

Видно, что смягчение модуля Юнга при охлаждении начинается с температур выше комнатной и достигает максимальной величины при $T_{\min } \approx 272 \mathrm{~K}$. Резкий минимум на кривой $\Delta E(T) / E_{0}$ характерен для структурных фазовых переходов, где температура перехода $T_{S}$ очень близка к $T_{\min }$ (точнее соответствует скачку на кривой $\Delta E(T) / E_{0}$ или максимуму производной модуля по температуре [15]). Зависимость $\Delta E(T) / E_{0}$ в области фазового перехода обнаруживает температурный гистерезис, такой что температуры скачка $T_{S d}$ при охлаждении и $T_{S u}$ при нагреве отличаются на величину порядка $7 \mathrm{~K}$. На зависимости $\Delta E(T) / E_{0}$ при нагреве наблюдается один выраженный минимум, тогда как при охлаждении имеется дополнительная аномалия в области $210-230 \mathrm{~K}$.

Фазовый переход в образце 1 сопровождается резким возрастанием поглощения от величины $2 \cdot 10^{-4}$ до $\sim 1.7 \cdot 10^{-2}$, причем поглощение остается большим и при $T \ll T_{S}$. Зависимости $q^{-1}(T)$ в области структурного перехода при нагреве и охлаждении также отличаются. При охлаждении коэффициент внутреннего трения $q^{-1}$ обнаруживает два максимума поглощения: слабый - при $T_{1} \sim T_{S d}$ и более сильный $\sim 1.7 \cdot 10^{-2}$ при более низкой температуре $T_{2}$, совпадающей с минимумом на зависимости $\Delta E(T) / E_{0}$. Максимум поглощения при более низкой температуре связан, по-видимому, с релаксацией структурных доменов. На упругие свойства при структурных фазовых переходах, сильное влияние могут оказывать структурные домены, возникающие ниже $T_{S}$. Под действием механических напряжений, обусловленных звуковой волной, происходит перестройка доменной структуры (например, длинные оси доменов выстраиваются вдоль растягивающего механического напряжения). Это эквивалентно дополнительной „мягкости“ в решетке и приводит к дальнейшему смягчению модуля $\Delta E(T) / E_{0}$ ниже $T_{S d}$. Такое поведение наблюдается, например, при структурных фазовых переходах янтеллеровской природы в редкоземельных цирконах [16]. Для исследованных сплавов минимальное значение модуля Юнга, достигается близко к температурам перехода $T_{S d}$ и $T_{S u}$, т. е. доменный вклад в модуль Юнга невелик. Видно, что как при охлаждении, так и при нагреве, более сильный максимум $q^{-1}(T)$ совпадает с минимумом на кривой $\Delta E(T) / E_{0}$. Более слабый максимум $q^{-1}(T)$ при нагреве близок к максимуму производной $d E / d T$.

Вторая аномалия на кривой $\Delta E(T) / E_{0}$ в области $210-230 \mathrm{~K}$, которая наблюдается только при охлаждении, также сопровождается возрастанием поглощения. Кривые $\Delta E(T) / E_{0}$ и $q^{-1}(T)$ при нагреве и охлаждении полностью совпадают ниже $\sim 210 \mathrm{~K}$ и выше $280 \mathrm{~K}$ (не показано на рис. 1). При этом с понижением температуры до $80 \mathrm{~K}$ упругий модуль линейно растет, а

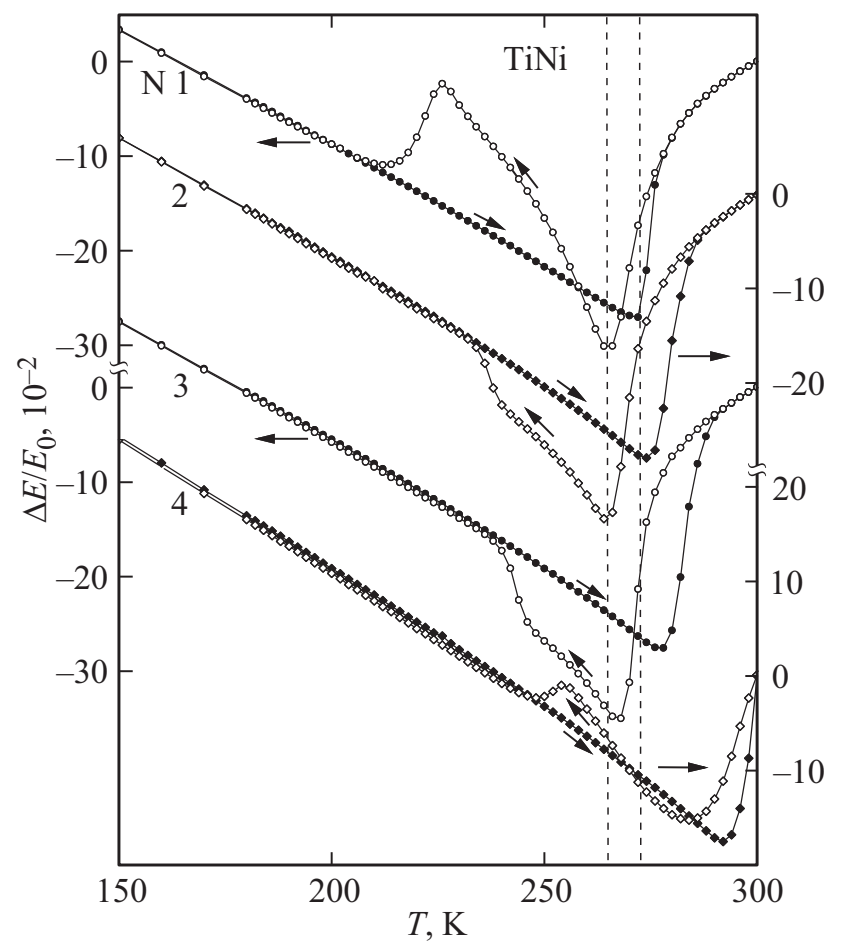

Рис. 2. Зависимость относительной величины модуля Юнга $\Delta E / E_{0}$ от температуры для образцов 1-4 сплава TiNi c различной термообработкой при нагреве (темные точки) и охлаждении (светлые точки; для образцов 1,3 - оси слева, для образцов 2,4 - оси справа).

поглощение немного уменьшается, оставаясь достаточно большим.

Сравнение кривых $\Delta E(T) / E_{0}$ для образцов $1-4$ позволяет проследить влияние термообработки на фазовый переход (рис. 2). Для закаленных образцов 2, 3 наблюдается качественно похожее поведение вблизи фазового перехода. Видно постепенное увеличение температуры $T_{S u}$ и более слабое увеличение температуры $T_{S d}$ от образца 1 к образцу 3, что приводит к увеличению гистерезиса. Для образца 4 температуры $T_{S d}$ и $T_{S u}$ лежит уже выше исследованного диапазона и о гистерезисе судить сложно. Таким образом температуры $T_{S d}$ для образцов практически совпадают и составляют $265 \pm 2 \mathrm{~K}$, а температуры $T_{S u}$ немного повышаются для образцов 2,3 . Температуры минимумов $T_{\min }$ на кривых при нагреве и охлаждении смещаются аналогичным образом. Величина смягчения модуля $\Delta E\left(T_{\min }\right) / E_{0}$ в минимуме при нагревании после термообработки практически не меняется, а при охлаждении величина смягчения $\Delta E\left(T_{\min }\right) / E_{0}$ увеличивается от образца 1 к образцу 3 .

Вне области структурного фазового перехода при $T<T_{S}$ зависимости $\Delta E(T) / E_{0}$ для образцов 2-4 также обнаруживают заметное отличие при нагреве и охлаждении. Для всех образцов на зависимости $\Delta E(T) / E_{0}$ при нагреве наблюдается один четкий минимум, тогда как при охлаждении имеется дополнительная аномалия 
в области $210-250 \mathrm{~K}$. При этом характер и знаки низкотемпературной аномалии для образца 1 до термообработки и образцов 2-3 после термообработки заметно отличаются. Интересно, что для отпущенного образца 4 дополнительная аномалия $\Delta E(T) / E_{0}$ при охлаждении качественно похожа на аномалию для образца 1 , только менее выражена и сдвинута, как и фазовый переход, в область высоких температур.

Более наглядно изменение температур фазового перехода $T_{S u}$ и $T_{S d}$ для образцов $1-4$ с различной термообработкой можно проследить на кривых производной $\left(1 / E_{0}\right) d E / d T$ (рис. 3). Максимумы на кривых, соответствующие скачкам модуля Юнга, смещаются в область более высоких температур, причем температура $T_{S u}$ смещается сильнее, чем температура $T_{S d}$. Отметим второй отрицательный пик на кривых $\left(1 / E_{0}\right) d E / d T$ в области $225-245 \mathrm{~K}$, который растет по величине и также смещается в область высоких температур от образца 1 к образцу 3. Второй положительный пик наблюдается только на образце 1 , который не подвергался дополнительной термообработке. Кривые $\left(1 / E_{0}\right) d E / d T$ при нагреве и охлаждении для образца 1 различаются в интервале температур 200-280 K, а для образцов 2, 3 этот интервал сужается.

На внутреннем трении $q^{-1}(T)$ для образцов $1-4$ также прослеживаются некоторые закономерности в зависимости от термообработки (рис. 4). На кривых

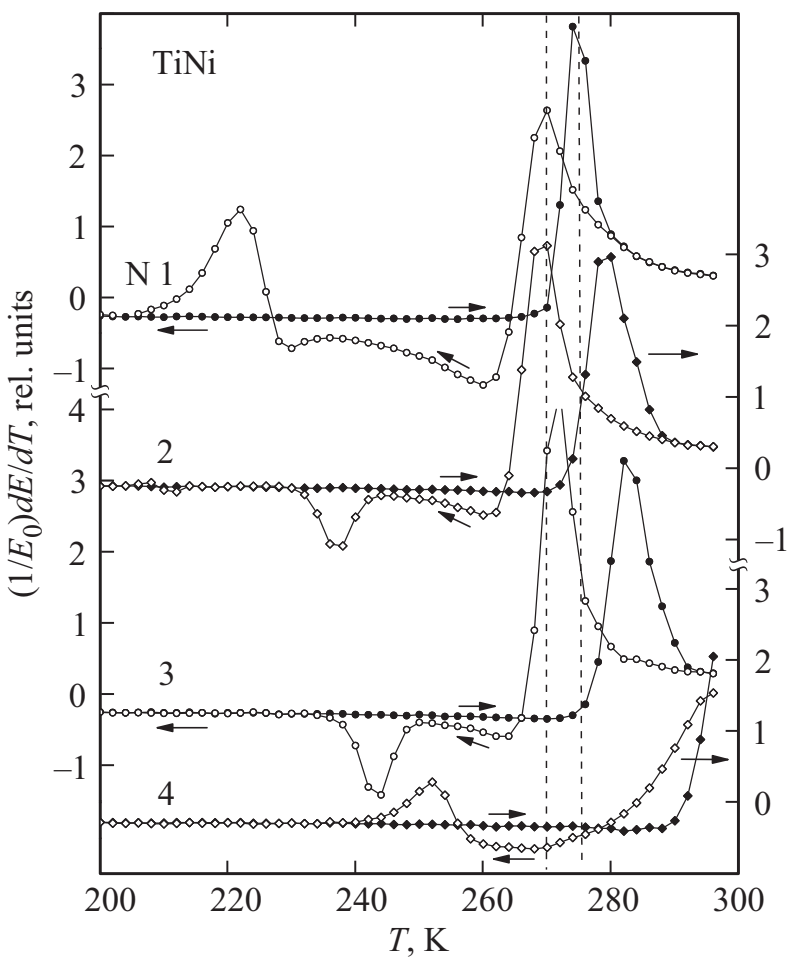

Рис. 3. Зависимость производной модуля Юнга $\left(1 / E_{0}\right) d E / d T$ от температуры для образцов 1-4 сплава TiNi с различной термообработкой при нагреве (темные точки) и охлаждении (светлые точки; для образцов 1,3 - оси слева, для образцов 2,4 - оси справа).

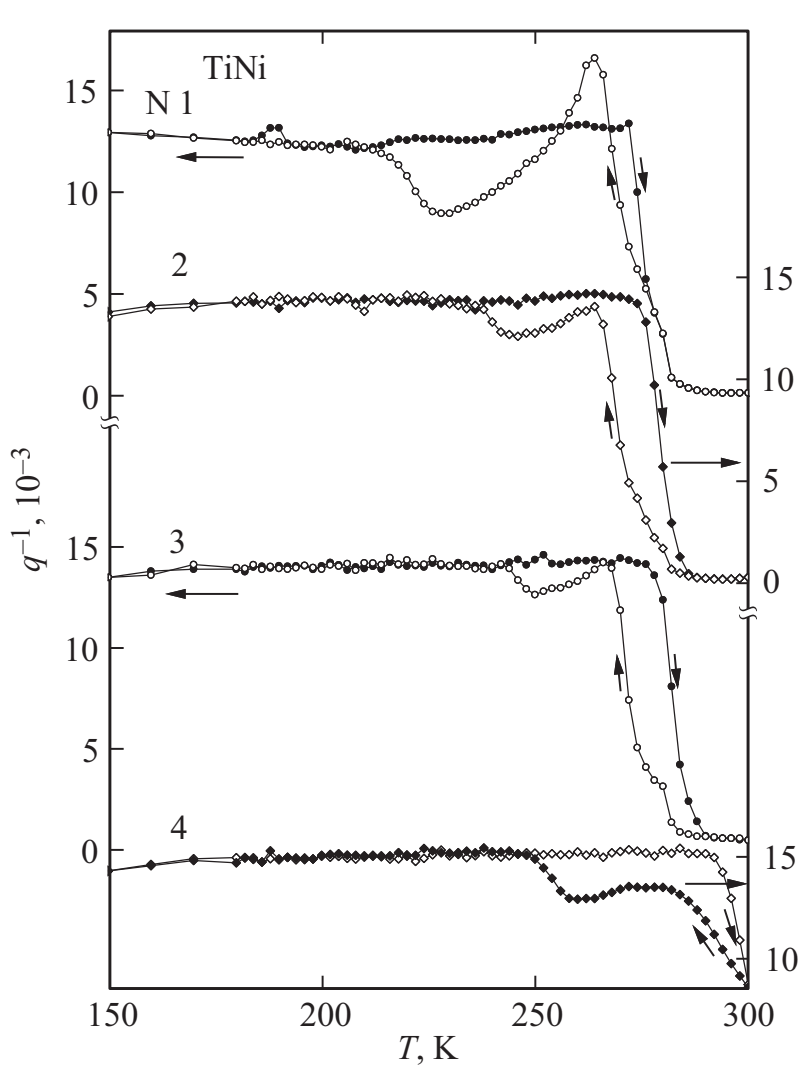

Рис. 4. Зависимость коэффициента внутреннего трения $q^{-1}(T)$ от температуры для образцов 1-4 сплава TiNi c различной термообработкой при нагреве (темные точки) и охлаждении (светлые точки; для образцов 1,3 - оси слева, для образцов 2,4- оси справа).

$q^{-1}(T)$ видно смещение температур $T_{S u}$ и $T_{S d}$ и увеличение гистерезиса. Отметим также изменение характера гистерезиса: для образца 1 кривые при охлаждении и нагреве начинают совпадать сразу при $T \geq T_{S}$, тогда как для образцов 2,3 различие сохраняется при $T$ заметно выше $T_{S}$. При этом максимум $q^{-1}(T)$ в области фазового перехода при охлаждении для образцов 2-4 уменьшается по величине и становится менее выраженным. Средний уровень поглощения при $T<T_{S}$ несколько увеличивается от $\sim 12 \cdot 10^{-3}$ до $\sim 16 \cdot 10^{-3}$.

\section{2. Фазовый переход аустенит-мартенсит в сплаве TiNi}

Постоянные упругой жесткости соединений, как известно, возрастают с понижением температуры вследствие ангармоничности колебаний кристаллической решетки. В области структурных фазовых переходов упругие постоянные испытывают сильные аномалии. Поведение модуля Юнга образца 1 сплава TiNi качественно согласуется с результатами акустических исследований на частоте $\sim 5 \mathrm{MHz}$ упругих свойств нитинола [3]. Некоторое отличие температур перехода может быть связано с отличием в составе образцов, а также микрострукту- 
ры, задаваемой термообработкой. Структурный фазовый переход с понижением симметрии решетки (изменением метрики решетки) сопровождается, как известно, обращением в ноль компоненты матрицы упругих модулей, сопряженной с деформацией („мягкая мода“) [17], а на других константах $c_{i j}$ аномалии существенно меньше $\left(\Delta c_{i j} / c_{i j} \sim 10^{-3}\right)$ [18]. Таким образом в этом случае зависимость модуля Юнга $E(T)$ отражает по существу изменение $\mathrm{c}$ температурой соответствующей мягкой моды $c_{i j}$.

Считается установленным, что эквиатомный состав нитинол $\mathrm{TiNi}$ обнаруживает каскад последовательных мартенситных превращений: высокотемпературная $\mathrm{TiNi}(\mathrm{I})$ фаза - промежуточная $\mathrm{TiNi}(\mathrm{II})$ фаза - низкотемпературные $\mathrm{TiNi}(\mathrm{III})$ и $\mathrm{TiNi}(\mathrm{IV})$ фазы. При исследовании нитинола в области температур 220-370 K наблюдаются два фазовых перехода. Резкое уменьшение модуля Юнга и модуля сдвига вблизи $300 \mathrm{~K}$ связывают с искажением решетки с сохранением объема и предмартенситным TiNi(II)/TiNi(III) превращением, обусловленным электрон-фононным взаимодействием. С другой стороны, аномалии упругих параметров вблизи $250 \mathrm{~K}$, которые свидетельствуют о деформации с изменением объема, связывают с мартенситным преобразованием $\mathrm{TiNi}(\mathrm{III})$ - TiNi(IV) с неупорядоченными комплексами.

Для исследованных нами сплавов нитинол TiNi смягчение модуля Юнга свидетельствует о неустойчивости кристаллической структуры при понижении температуры, а глубокие минимумы и последующий рост на зависимостях $\Delta E(T) / E_{0}$ обусловлены структурными фазовыми переходами. Для всех образцов температуры перехода $T_{S}$ очень близки, а смягчение модуля Юнга и скачок при фазовом переходе достигают величины $\sim 20-30 \%$. Величина смягчения $\Delta E(T) / E_{0}$ и максимум $q^{-1}$ в исследуемых образцах такого же порядка, что и в Р3 цирконах. Для кристаллического образца $\mathrm{DyVO}_{4}$, например, упругий модуль $\left(c_{11}-c_{12}\right)$ при ян-теллеровском переходе $B_{1 g}$-типа обнаруживает смягчение до нуля, а смягчение модуля поликристаллического образца составляет $\Delta E(T) / E_{0} \approx 30 \cdot 10^{-2}[16]$. Таким образом для нитинола при предмартенситном переходе можно предполагать обращение в ноль одной из компонент матрицы упругих модулей. Для структурного перехода от кубической В2 фазы к тетрагональной или более низкосимметричной фазе ожидается смягчение модуля $\left(c_{11}-c_{12}\right)$ или $c_{44}$ в зависимости от оси деформации [17].

В основном характер зависимостей для трех образцов с разной термообработкой повторяется. Точка предмартенситного перехода, аномалия в этой точке и гистерезис воспроизводятся. Однако, мартенситный переход при температуре $\sim 230 \mathrm{~K}$ для образцов, отличающихся термообработкой, проявляется по-разному, например различаются величины, знаки и характер аномалий. Для образца 1 сложное поведение $\Delta E(T) / E_{0}$ можно связать с двух ступенчатым характером перехода аустенитмартенсит, когда небольшая доля образца „задерживается““ в высокотемпературной фазе до температуры потери устойчивости фазы. Отличие в поведении $\Delta E(T) / E_{0}$ для образцов с различной термообработкой отражает, на наш взгляд, отличие в их микроструктуре/дефектности, влияющей на кинетику перехода, например образование зародышей низкотемпературной фазы при фазовом переходе.

\section{4. Заключение}

В настоящей работе проведены сравнительные исследования упругих характеристик коммерческих сплавов нитинол TiNi c памятью формы с различной термообработкой, в интервале температур 80-300 К, включающем предмартенситный и мартенситный переходы. Смягчение модуля Юнга исследованных сплавов при понижении температуры свидетельствует о неустойчивости кристаллической структуры, а глубокие минимумы и последующий рост на зависимостях $\Delta E(T) / E_{0}$ обусловлены предмартенситным переходом при $T_{S} \approx 270 \mathrm{~K}$. Значительное смягчение $\sim 20-30 \%$ модуля Юнга при предмартенситном переходе позволяет предполагать обращение в ноль одной из компонент матрицы упругих модулей. При дополнительной термообработке температуры фазового перехода $T_{S u}$ при нагреве и $T_{S d}$ при охлаждении немного смещаются и гистерезис увеличивается, а характер упругих аномалий практически не меняется. Наоборот, для мартенситного перехода при $T \sim 230 \mathrm{~K}$ аномалия упругих свойств наблюдаются только при охлаждении, а температура, величина и характер аномалии претерпевают значительные изменения после термообработки. Сложное поведение $\Delta E(T) / E_{0}$ при охлаждении можно связать с двух ступенчатым характером перехода аустенит-мартенсит, когда небольшая доля образца „задерживается“ в высокотемпературной фазе до температуры потери устойчивости фазы. Отличие в поведении $\Delta E(T) / E_{0}$ образцов отражает, на наш взгляд, отличие в их микроструктуре/дефектности, влияющей на кинетику перехода, например образование зародышей низкотемпературной фазы при фазовом переходе.

\section{Конфликт интересов}

Авторы заявляют, что у них нет конфликта интересов.

\section{Список литературы}

[1] Ю.Н. Коваль, В.А. Лободюк. Успехи физ. мет. 7, 53 (2006).

[2] F.E. Wang, B.F. Desavage, W.J. Buehler. J. Appl. Phys. 39, 2166 (1968).

[3] M. Fukuhara, M. Yagi, A. Matsuo. Phys. Rev. B 65, 224210 (2002).

[4] F.E. Wang, W.J. Buehler, S.J. Pickart. J. Appl. Phys. 36, 3232 (1965).

[5] F.E. Wang, S.J. Pickart, H.A. Alperin. J. Appl. Phys. 43, 97 (1972).

[6] M. Arciniegas, J.M. Manero, J. Pen A, F.J. Gil, J.A. Planell. Metal. Mater. Ttrans. A 39, 742 (2008). 
[7] W.M. Huang, H.K. Lim. J. Mater. Sci. Lett. 22, 1399 (2003).

[8] V.G. Pushin, N.N. Kuranova, A.V. Pushin, A.N. Uksusnikov, N.I. Kourov, T.E. Kuntsevich. Fizika Metallov i Metallovedenie 117, 1302 (2016).

[9] N.N. Kuranova, A.V. Pushin, V.G. Pushin, N.I. Kourov. Fizika Metallov i Metallovedenie 119, 618 (2018).

[10] С В. Разоренов, Г.В. Гаркушин, Г.И. Канель, О.А. Кашин, И.В. Раточка. ФТТ 53, 768 (2011).

[11] M. Morinaga, N. Yukawa. In: Computer Aided Innovation of New Materials / Eds M. Doyama, T. Suzuki, J. Kihara, R. Yamamoto. Elsevier Science Publisher B., North-Holland (1991). P. 803.

[12] H. Tripathy, S. Raju, R.N. Hajra, S. Saibab. Met. Mater. Ttrans. A 49, 979 (2018).

[13] A. Wolfenden, M.D. Compere. J. Mater. Sci. Lett. 12, 1128 (1993).

[14] M. Fukuhara, A. Sanpei. Phys. Rev. B 49, 13099 (1994).

[15] L.R. Testardi. Phys. Rev. B 12, 3849 (1975).

[16] А.Е. Дворникова, 3.А. Казей, В.И. Соколов. ЖЭТФ 96, 1444 (1989).

[17] C. Jaussaud, P. Morin, D. Schmitt. J. Magn. Magn. Mater. 22, 98 (1980).

[18] G.A. Gehring, E.A. Gehring. Rep. Prog. Phys. 38, 1 (1975).

Редактор К.В. Емцев 